\title{
APPLICATIONS OF DENSITY MATRIX RENORMALIZATION GROUP TO PROBLEMS IN MAGNETISM
}

\author{
G.A. Gehring, R.J. BursiLL*
}

Department of Physics, The University of Sheffield, Sheffield, S3 7RH, United Kingdom

\author{
AND T. XIANG
}

Interdisciplinary Research Centre in Superconductivity, The University of Cambridge Cambridge, CB3 0HE, United Kingdom

The application of real space renormalization group methods to quantum lattice models has become a topic of great interest following the development of the density matrix renormalization group by White. This method has been used to find the ground and low-lying excited state energies and wave functions of quantum spin models in which the form of the ground state is not clear, for instance because the interactions are frustrated. It has also been applied to fermion problems where the tendency for localization due to the strong Coulomb repulsion is opposed by the lowering of the kinetic energy which occurs as a result of electron transfer. The approach is particularly suitable for one-, or quasi-one-dimensional problems. The method involves truncating the Hilbert space in a systematic and optimised manner. Results for the ground state energy are thus variational bounds. The results for low-lying energies and correlation functions for one-dimensional systems have unprecedented accuracy and the method has become the method of choice for solving one-dimensional quantum spin problems. We review the method and results obtained for the spin-1 chain with biquadratic exchange as well as the spin-1/2 model with competing nearest and next nearest neighbour exchange will be described. More recently, the density matrix renormalization group has been applied to reformulate the coupling constant renormalization group approach which is appropriate for the study of critical properties. This approach has been applied to the anisotropic spin-1/2 Heisenberg chain. Finally, we discuss recent work which has borne promising applications in two dimensions - the Ising model and the two-dimensional Hubbard model.

PACS numbers: 11.10.Gh, 64.60.-i

*Present address: School of Physics, UNSW, Sydney, 2052, Australia. 


\section{Introduction}

Recent times have seen the fabrication of an increasingly rich variety of materials whose low energy magnetic properties require intrinsically quantum mechanical as well as low-dimensional lattice models for a realistic description. On the other hand, the development of sophisticated field theories and the surprising conjecture of Haldane [1] has dramatically rekindled theoretical and mathematical interest in one-dimensional quantum spin models. Also, the advent of high temperature superconductors has generated intensive interest in the magnetic properties of two-dimensional quantum lattice models such as the Hubbard, $t-J$ and Heisenberg models.

It is well known that analytical solutions of these models are only available in rare and special cases and that mean field theories perform unreliably when applied to low-dimensional systems. Also, the presence, for example, of frustration in these systems renders the construction of suitable variational wave functions very difficult as the structure of the ground state is not at all clear ahead of time. As a result, perturbative attempts at systematically improving upon variational model states are often prone to slow or even spurious convergence. This problem of frustration has also for a long time plagued attempts at applying Monte Carlo methods to many of the above-mentioned systems at low temperatures. Exact diagonalization approaches may fail to reach suitably large lattices for two-dimensional or coupled chain problems due to the exponential growth in the size of the Hilbert space. This is especially problematic for frustrated systems which possess incommensurate order in the ground state.

There has therefore been a real window of opportunity open for the development of a portable, robust and systematically improvable numerical scheme for solving low-dimensional quantum lattice models of magnetic systems. The advent of the renormalization group (RG) [2] and Wilson's highly successful numerical solution of the Kondo problem [2] in addition to promising applications to classical systems in two dimensions [3] suggested that the real space renormalization group (RSRG) might well fill this rôle.

Unfortunately, attempts at carrying out this programme produced results with disappointingly slow convergence [4]. The underlying causes for the poor performance of the RSRG were addressed in a seminal series of papers by White [5] where a powerful improvement on the RSRG, the density matrix renormalization group (DMRG), was formulated. These papers were followed with a phenomenally successful study of the spin-1 chain where the Haldane conjecture, that spin chains of integral spin are gapped, was verified with unprecedented accuracy [6]. A flood of applications to problems in low-dimensional quantum magnetism has since followed.

In this article we review the DMRG and some its applications to problems in magnetism. In Sec. 2 we briefly outline the method and the quantities of interest which can be calculated. In Sec. 3 we discuss applications of the DMRG to spin chains with frustration. In Sec. 4 we review attempts at using the DMRG to study phase transitions in these systems, either by direct calculation of order parameters or by deriving RG transformations in the space of coupling constants. 
In Sec. 5 we discuss recent extensions of the DMRG to two-dimensional problems and one-dimensional models at non-zero temperature.

\section{The DMRG method}

The DMRG is an iterative, truncated basis procedure whereby a large system (or superblock) is built up from a small number of lattice sites by adding a few sites at a time. As mentioned, the DRMG was introduced and formally developed in a series of papers [5] by White and co-workers at Irving. We refer the reader to these excellent papers for a detailed description of the method. Here we will attempt to present a simple exposition which captures the essential algorithmic steps involved. It will be useful to work with a concrete model; the one-dimensional, spin-1/2 $X Y$ model

$$
\mathcal{H}=\sum_{i}\left(S_{i}^{+} S_{i+1}^{-}+\text {h.c. }\right),
$$

where $S_{i}^{ \pm}$is the spin-1/2 raising (lowering) operator for lattice site $i$.

Let us initially define a system block $A$ which consists of only a single site and hence has $M=2$ states $|1\rangle=|\uparrow\rangle$ and $|2\rangle=|\downarrow\rangle$. We record the matrix elements of the spin operator $S_{A}^{+}$for this site, as well as the block Hamiltonian $\mathcal{H}_{A}$, with respect to the system block basis $\{|n\rangle: n=1, \ldots, M\}$ i.e. $S\left(n^{\prime}, n\right) \equiv\left\langle n^{\prime}\left|S_{A}^{+}\right| n\right\rangle$ and $H\left(n^{\prime}, n\right) \equiv\left\langle n^{\prime}\left|\mathcal{H}_{A}\right| n\right\rangle \equiv 0$.

In the first iteration we form an environment block $B$ which is precisely the same as the system block (a single site). Combining the system block, the environment block and two extra sites, we form a superblock $A \bullet \bullet B$. A basis for the superblock is given by

$$
\left\{\left|n_{S}\right\rangle \equiv\left|n_{A}\right\rangle \otimes\left|s_{1}\right\rangle \otimes\left|s_{2}\right\rangle \otimes\left|n_{B}\right\rangle: n_{A}, n_{B}=1, \ldots, M ; s_{1}, s_{2}=\uparrow, \downarrow\right\} .
$$

We write down the Hamiltonian $\mathcal{H}_{S} \equiv \mathcal{H}_{A}+\mathcal{H}_{A \bullet}+\mathcal{H}_{\bullet \bullet}+\mathcal{H}_{\bullet B}+\mathcal{H}_{B}$ for the superblock viz.

$$
\begin{aligned}
& \left\langle n_{S}^{\prime}\left|\mathcal{H}_{A}\right| n_{S}\right\rangle=\delta_{s_{1}^{\prime} s_{1}} \delta_{s_{2}^{\prime} s_{2}} \delta_{n_{B}^{\prime} n_{B}} H\left(n_{A}^{\prime}, n_{A}\right), \\
& \left\langle n_{S}^{\prime}\left|\mathcal{H}_{A \bullet}\right| n_{S}\right\rangle=\delta_{s_{2}^{\prime} s_{2}} \delta_{n_{B}^{\prime} n_{B}}\left[S\left(n_{A}^{\prime}, n_{A}\right)\left\langle s_{1}^{\prime}\left|S_{\bullet}^{-}\right| s_{1}\right\rangle+\text { h.c. }\right] .
\end{aligned}
$$

Low energy eigenstates of the 4-site Hamiltonian $\mathcal{H}_{S}$ may be found at this point.

Next, we expand the system block by augmenting it with one of the extra sites. A basis for the augmented block $\widetilde{A} \equiv A \bullet$ is given by

$$
\left.\{|n\rangle\rangle \equiv\left|n_{A}\right\rangle \otimes\left|s_{1}\right\rangle: n_{A}=1, \ldots, M ; s_{1}=\uparrow, \downarrow\right\}
$$

and clearly has $\widetilde{M} \equiv 2 M$ states.

In order to again form a (larger, by two sites) superblock, we require the matrix elements of the augmented block Hamiltonian $\mathcal{H}_{\tilde{A}} \equiv \mathcal{H}_{A}+\mathcal{H}_{A}$ as well as the spin operator corresponding to the added (end) site viz.

$$
\begin{aligned}
& \widetilde{H}\left(n^{\prime}, n\right) \equiv\left\langle\left\langle n^{\prime}\left|\mathcal{H}_{\widetilde{A}}\right| n\right\rangle\right\rangle \\
& \quad=\delta_{s_{1}^{\prime} s_{1}} H\left(n_{A}^{\prime}, n_{A}\right)+\left[S\left(n_{A}^{\prime}, n_{A}\right)\left\langle s_{1}^{\prime}\left|S_{\bullet}^{-}\right| s_{1}\right\rangle+\text { h.c. }\right], \\
& \widetilde{S}\left(n^{\prime}, n\right) \equiv\left\langle\left\langle n^{\prime}\left|S_{\bullet}^{+}\right| n\right\rangle\right\rangle \\
& \quad=\delta_{n_{A}^{\prime} n_{A}}\left\langle s_{1}^{\prime}\left|S_{\bullet}^{+}\right| s_{1}\right\rangle .
\end{aligned}
$$


The superblock construction (2), (3) and (4) can now be extended indefinitely where $\widetilde{A} \mapsto A, \widetilde{H} \mapsto H, \widetilde{S} \mapsto S, \widetilde{M} \mapsto M$ and $|\rangle.\rangle \mapsto|$.$\rangle . However, even with the$ aid of sparse matrix diagonalization methods, the size of the superblock basis, $\mathrm{O}\left(M^{2}\right)$ will soon grow prohibitively large as $M$ is doubled at each iteration.

Instead, we truncate the basis of the augmented block so that the number of states used in the subsequent iteration does not exceed a fixed threshold, $m$. The truncated basis is chosen so as to contain the most important states for forming low energy eigenstates of the $\mathcal{H}_{S}$. White's most important innovation was the use of a density matrix to determine these states.

For instance, if we are interested in the ground state $\left|\psi_{\mathrm{G}}\right\rangle$, then we form a reduced density matrix $\rho$ for the augmented block as follows:

$$
\left\langle\left\langle n^{\prime}|\rho| n\right\rangle\right\rangle \equiv \sum_{s_{2}, n_{B}}\left\langle n_{S}^{\prime} \mid \psi_{\mathrm{G}}\right\rangle\left\langle\psi_{\mathrm{G}} \mid n_{S}\right\rangle
$$

with

$$
\begin{aligned}
& \left.\left|n_{S}\right\rangle \equiv\left|n_{A}\right\rangle \otimes\left|s_{1}\right\rangle \otimes\left|s_{2}\right\rangle \otimes\left|n_{B}\right\rangle \equiv|n\rangle\right\rangle \otimes\left|s_{2}\right\rangle \otimes\left|n_{B}\right\rangle \\
& \left.\left|n_{S}^{\prime}\right\rangle \equiv\left|n_{A}^{\prime}\right\rangle \otimes\left|s_{1}^{\prime}\right\rangle \otimes\left|s_{2}\right\rangle \otimes\left|n_{B}\right\rangle \equiv\left|n^{\prime}\right\rangle\right\rangle \otimes\left|s_{2}\right\rangle \otimes\left|n_{B}\right\rangle .
\end{aligned}
$$

That is, the superblock ground state projection operator is traced over the environmental degrees of freedom $\bullet B$.

The density matrix eigenstates $\{|n\rangle\rangle\rangle: n=1, \ldots, \widetilde{M}\}$ are found by standard dense matrix diagonalization methods and the $m$ most important states (corresponding to the $m$ largest eigenvalues of $\rho$ ) are retained in forming the system block for the next iteration. Of course, the operators of interest must be represented in terms of these states viz.

$$
\tilde{H}\left(n^{\prime}, n\right) \mapsto \sum_{n^{\prime \prime} n^{\prime \prime \prime}}\left\langle\left\langle\left\langle n^{\prime} \mid n^{\prime \prime}\right\rangle\right\rangle \widetilde{H}\left(n^{\prime \prime}, n^{\prime \prime \prime}\right)\left\langle\left\langle n^{\prime \prime \prime} \mid n\right\rangle\right\rangle\right\rangle \mapsto H\left(n^{\prime}, n\right),
$$

where $1 \leq n, n^{\prime} \leq \min \{\widetilde{M}, m\} \mapsto M$.

The density matrix eigenvalues sum to unity and the truncation error, defined as the sum of the density matrix eigenvalues corresponding to discarded eigenvectors gives a qualitative indication as to the accuracy of the calculation as well as providing a framework for extrapolation to the $m=\infty$ limit [6]. As a result of basis truncation, the cpu time is linear in the superblock size and so one can easily proceed to the thermodynamic limit for intrinsic properties such as the ground state energy density (see [7] for a formal discussion of the DMRG fixed point and the thermodynamic limit of the DMRG).

As mentioned, the accuracy of results obtained in this way was unprecedented [6], the accuracy of the ground state energy density $e_{0}$ for the spin-1 chain being ultimately limited by the precision of machine arithmetic viz. $e_{0} \approx$ $-1.401484038971(4)$. Similarly exquisite accuracy persists when targeting low-lying excitation energies in various symmetry sectors $[6]$ e.g. $E_{1}-E_{0} \approx 0.41050(2)$.

The method described above is the infinite lattice algorithm. In the finite lattice algorithm, the infinite lattice algorithm is initially applied so as to obtain a superblock of a desired size. System blocks are retained at all iterations and are used as environment blocks for the purpose of recursively deriving progressively 
superior system blocks within the superblock whose size remains fixed [5]. Repeated sweeps across the lattice result in substantial improvements in accuracy, especially for the study of edge states and systems with impurities.

Applications to spin chains with high spin $[8,9]$, dimerization and/or frustration [10-15], together with extensions to coupled spin chains [16], models with itinerant fermions [17,18], Kondo systems [19-22], as well as coupled fermion chains [23] have followed, even in cases of intermediate doping. Formulations for systems with single [24] as well as randomly distributed [25] impurities and disorder [26] have also been forthcoming.

By retaining matrix elements of operators acting on sites throughout the blocks, it is possible to calculate static correlation functions. In Refs. [6] and [27] it is shown that highly accurate correlation functions can be calculated over many lattice spacings. Highly accurate studies of the structure factor and string order parameter (topological long-range order) [6], as well as edge states [28] in Haldane phase systems have also been performed. Dynamical correlation functions have also been calculated within the static [29], continued fraction [30] and correction vector [31] approaches. Finally, the DMRG has been formulated for the solution of models of spin chains dynamically coupled to (dispersionless) phonons [32].

\section{Spin chains with frustration}

A spin model on a bipartite lattice with a nearest neighbour, antiferromagnetic Heisenberg interaction can have its tendency towards commensurate order in the ground state frustrated in the presence of other interactions. In this section we consider two such models in one dimension. In each case, $J_{1}>0$ denotes the strength of the Heisenberg interaction and $J_{2} \geq 0$ - the strength of the frustrating term.

The Majumda-Gosh (MG) model [33] has the Heisenberg interaction frustrated by the presence of next-neighbour interactions viz.

$$
H=\sum_{i}\left[J_{1} S_{i} S_{i+1}+J_{2}^{(\mathrm{MG})} S_{i} S_{i+2}\right]
$$

where $S_{i}$ denotes the spin $S=1 / 2$ operator for lattice site $i$. This model has some relevance to the interpretation of experiments on quasi one-dimensional spin- $1 / 2$ chains $[34,35]$. For $S=1$, the order can also be disturbed in the Lai-Sutherland (LS) [36] model through the presence of a biquadratic term

$$
H=\sum_{i}\left[J_{1} S_{i} S_{i+1}+J_{2}^{(\mathrm{LS})}\left(S_{i} S_{i+1}\right)^{2}\right] .
$$

The models (12) $[33-35,37]$ and $(13)[36,38,39]$ have been the subject of intense study in the past. Exact diagonalization methods together with exact solutions and rigorous results at special points have been combined to give a good picture of their critical properties.

In the MG case, the pure Heisenberg point $\left(J_{2}=0\right)$ has been extensively studied [40] and is a gapless spin liquid with quasi long-range order (algebraically decaying correlations). This behaviour persists up to a critical point [37] $J_{2 \mathrm{c}} \approx$ $0.241167 J_{1}$ where a Kosterlitz-Thouless (KT) transition occurs to a gapped, dimer phase with exponentially decaying correlations. A simple dimer wave function 
becomes exact at the MG point $\left(J_{2}=J_{1} / 2\right)$ [33]. Here it can be shown that the gap is non-zero [41]. At $J_{2}=\infty$, the lattice separates into two uncoupled Heisenberg chains and is thus again critical.

For the LS model, the Heisenberg point is not critical, there being a non-zero gap and exponentially decaying correlations $[1,6]$. At the Affleck point $\left(J_{2}=J_{1} / 3\right)$ a simple valence bond solid (VBS) wave function provides the exact ground state [41] and the model is rigorously known to be gapped [41]. The model is solvable at the LS point $\left(J_{2}=J_{1}\right)$ [36], having soft mode excitations at $k= \pm 2 \pi / 3$. The best numerical studies [38] indicate that this point is critical (i.e. $J_{2 \mathrm{c}}=J_{1}$ ), separating a gapped VBS (Haldane) phase from a gapless phase with a quasi long-ranged 3-fold ground state periodicity which persists up to the ferromagnetic boundary $\left(J_{2}=\infty\right)$, the transition again being of the KT type.

Until the advent of the DMRG, less was understood regarding the nature of incommensurate correlations in these models. In the classical limit $S=\infty$, these models both exhibit a crossover to incommensurate behaviour at some threshold $J_{2 \mathrm{cl}}$. For the MG model we have $J_{2 \mathrm{cl}}=J_{1} / 4$ and $J_{2 \mathrm{cl}}=J_{1} / 2$ for the LS model. For $J_{2} \leq J_{2 \mathrm{cl}}$ correlations are commensurate, spins being antialigned in a Néel ground state. For $J_{2}>J_{2 \mathrm{cl}}$ the classical pitch angle $\theta_{\mathrm{cl}}$ between successive spins is given by $\theta_{\mathrm{cl}} \equiv \cos ^{-1}\left(-J_{2 \mathrm{cl}} / J_{2}\right)$. The DMRG has allowed a characterization of the incommensurate behaviour through the accurate calculation of correlations over large distances within large chains.

In a series of studies of the structure factor

$$
S(k) \equiv \frac{1}{N} \sum_{j, j^{\prime}=1}^{N} C_{j j^{\prime}} \mathrm{e}^{\mathrm{i}\left(j-j^{\prime}\right) k},
$$

where

$$
C_{j j^{\prime}} \equiv\left\langle\psi_{\mathrm{G}}\left|S_{j}^{z} S_{j^{\prime}}^{z}\right| \psi_{\mathrm{G}}\right\rangle
$$

is the correlation function and $N$ - the lattice size, a threshold for the onset of incommensurate correlations $J_{2 L}$ was defined as the value of $J_{2}$ at which the peak in the structure factor $k_{\max }$ begins to deviate from $\pi$ [10-13]. Plots of $k_{\max }$ as well as $\theta_{\mathrm{cl}}$ for the two models are given in Fig. 1.

The DMRG permits accurate pinpointing of the threshold. For the MG model we have $J_{2 \mathrm{~L}} \approx 0.5206 J_{1}[12]$ and for the LS model $J_{2 \mathrm{~L}} \approx 0.4684 J_{1}$ [10]. Schollwöck et al. [11] studied $C(r)$, the correlation in real space. They observed the existence of a disorder point $J_{2 \mathrm{D}}$ marking the onset of incommensurability in real space. They interpreted $J_{2 \mathrm{~L}}$ as being a Lifshitz point. In fact, it was shown for the LS model that $J_{2 \mathrm{D}}=J_{1} / 3$, i.e. the disorder point was precisely the Affleck point. Bursill et al. [12] showed that for the MG model, $J_{2 \mathrm{D}}=J_{1} / 2$, i.e. the disorder point coincides with the $M G$ point. $J_{2 D}$ lies in the gapped phase, near to where the gap in maximal $[11,35]$. Numerically it appears as though the correlation length $\xi$ takes on its minimum at $J_{2}=J_{2 \mathrm{D}}$. Schollwöck et al. have pointed out that $J_{2 \mathrm{cl}}, J_{2 \mathrm{~L}}$ and $J_{2 \mathrm{D}}$ should be expected to merge in the classical limit $S \rightarrow \infty$. 


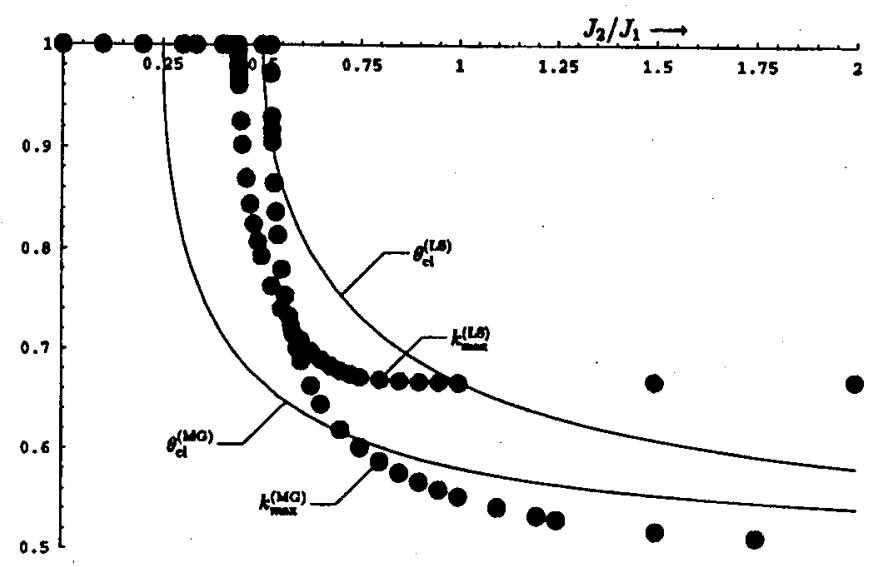

Fig. 1: DMRG results for the structure factor peak position $k_{\max }$ and classical pitch angle $\theta_{c l}$ (in units of $\pi$ ) as functions of the frustration $J_{1} / J_{2}$ for two frustrated spin systems - the MG and LS models.

\section{The DMRG and critical phenomena}

A critical point in a quantum lattice model is a point in the parameter space at which the ground state energy is non-analytic. Generally, the energy gap $\Delta$ (between the ground and first excited states) vanishes as the critical point is approached and, correspondingly, the correlation length $\xi$ diverges.

As a result of the long-range correlations in the vicinity of critical points, attempts to characterise them using exact diagonalization methods may be hindered by limitations on the system sizes which can be reached. In principle, the DMRG can study very large systems with high accuracy and so it is natural to apply the DMRG to the study of critical points. In this section we review attempts to do so in magnetic models. We distinguish between methods which characterise critical points via direct calculations of $\Delta$ or $\xi$, and those which do so by generating renormalization group transformations in the space of coupling constants.

\subsection{Direct calculations of $\Delta$ or $\xi$}

The direct calculation of $\Delta$ or $\xi$ in the vicinity of a critical point within the DMRG is hindered by the fact that the convergence of calculated eigenvalues or correlation functions with $m$ is slowest when $\Delta$ is small or $\xi$ is large $[5,10,11]$. Nevertheless, such calculations have been forthcoming.

Chitra et al. [13] have calculated $\Delta$ for the MG model (12) and $\xi[10,11]$ and $\Delta[11]$ have been calculated for the LS model (13) but in all cases, though high accuracy is possible near the disorder point $J_{2}=J_{2 \mathrm{D}}$, results become too inaccurate as the critical point $J_{2 \mathrm{c}}$ is approached for any recovery of the finite size scaling results of [37] and [38].

For the MG model, Bursill et al. [12] have also defined a dimer order parameter

$$
D \equiv \lim _{N \rightarrow \infty}\left|C_{N / 2-1} N / 2-C_{N / 2 N / 2+1}\right|
$$




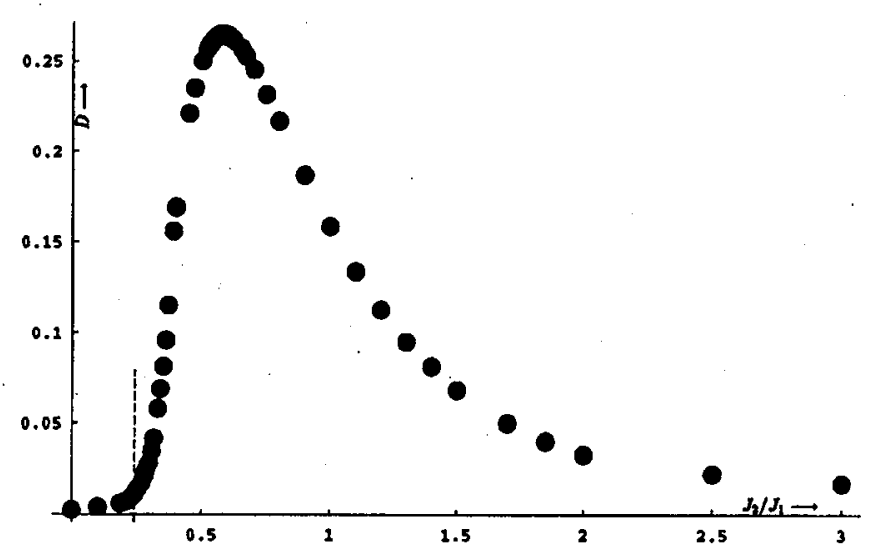

Fig. 2. DMRG results for the dimer order parameter $D$ as a function of the frustration $J_{1} / J_{2}$ for the MG model. The known position of the phase transition is indicated by the dashed line.

which characterizes the dimer phase $J_{2 \mathrm{c}}<J_{2}<\infty$, vanishing as $J_{2} \rightarrow\left(J_{2 \mathrm{c}}\right)^{+}$. A plot of $D$ versus $J_{2} / J_{1}$, calculated with $m=200$ is given in Fig. 2. Again, the critical point is not pinpointed with this approach.

These somewhat disappointing results are not surprising given that the phase transitions are of the KT type, from a gapped phase to an extended gapless phase with an essentially singular divergence in $\xi$ which is extremely difficult to recover numerically without recourse to scaling theory.

For transitions such as the Ising transition where the order parameter vanishes only at the critical point and the system is gapped elsewhere, there is more hope for direct approaches. The exactly solvable $S=1 / 2$ transverse Ising (ITF) model

$$
\mathcal{H}=-\gamma \sum_{i} S_{i}^{z}-\sum_{i} S_{i}^{x} S_{i+1}^{x}
$$

has an Ising transition at $\gamma_{c}=2$ which is accurately recovered by the direct approach [42].

Kato and Tanaka [14] have used the DMRG to pinpoint such a transition in the dimerised $S=1$ chain

$$
\mathcal{H}=\sum_{i}\left(1-(-1)^{i} \delta\right) S_{i} S_{i+1}
$$

at $\delta=0.25 \pm 0.01$. Also, Pati et al. [15] appear to have discovered such a point in the $S=1$ case of (12) at $J_{2}=0.730 \pm 0.05$. Ladder models consisting of two coupled Heisenberg chains have been studied [16]. The phase transition whereby a gap opens on the introduction of interchain exchange, has been characterised.

Finally, by implementing new efficiency measures into the finite lattice algorithm, White and Affleck [35] have performed calculations on the MG model (12) for lattices with many thousands of sites using large values of $m$. These calculations afford an accurate study of $k_{\max }, \xi$ and $\Delta$ for relatively large values of $J_{2}$. 
With the help of field theory, this allows a characterization of the critical point at $J_{2}=\infty$.

\subsection{Coupling constant transformations from the DMRG}

As mentioned, critical points and exponents can be calculated by generating $R G$ transformations in the space of coupling constants. This approach has been applied extensively using the RSRG to derive the transformations [43].

The idea is that a system of $N$ sites, with Hamiltonian $\mathcal{H}$ parameterised by coupling constant(s) $\gamma$, is divided into $N / b$ blocks of $b$ sites. $N / b$-fold tensor products of the $m$ lowest energy eigenstates

$$
B \equiv\left\{\left|\psi_{i}\right\rangle: i=1, \ldots, m\right\}
$$

of the block Hamiltonians are used to form a truncated basis $B \otimes \ldots \otimes B$ (of dimension $m^{N / b}$ ) for the system. $B$ is then identified as being a basis for a small repeat unit in a lattice model - a single site, or a multisite. For example, if $m=2$ then $B$ is identified as the basis for a site containing an $S=1 / 2$ operator, if $m=4$ then identification with a multisite consisting of two $S=1 / 2$ atoms is appropriate. $\mathcal{H}^{\prime}$, the projection of $\mathcal{H}$ onto $B \otimes \ldots \otimes B$ can then be identified with a spin Hamiltonian.

If $\mathcal{H}^{\prime}$ is of the same form as $\mathcal{H}$, but with a different constant (vector) $\gamma^{\prime}$ then

$$
\gamma \mapsto \gamma^{\prime}=T(b \mid \gamma)
$$

constitutes a RG transformation from which critical points

$$
\gamma_{c}=T\left(b \mid \gamma_{c}\right)
$$

and critical exponents such as the thermal exponent

$$
\nu=\frac{\log b}{\log \left|T^{\prime}\left(b \mid \gamma_{c}\right)\right|}
$$

can be extracted.

The RSRG results for the ITF model were quite acceptable [44]. However, for the anisotropic $S=1 / 2$ Heisenberg $(X X Z)$ model

$$
\mathcal{H}=\sum_{i}\left[S_{i}^{z} S_{i+1}^{z}+\frac{\gamma}{2}\left(S_{i}^{+} S_{i+1}^{-}+\text {h.c. }\right)\right]
$$

which is essentially singular $(\nu=\infty)$ at the Heisenberg point $\left(\gamma_{c}=1\right)$, the RSRG result was incorrect [45] in that $\nu \downarrow 2$ as $b \rightarrow \infty$.

It is natural to apply the DMRG philosophy to generate coupling constant transformations. That is, instead of using the $m$ lowest eigenstates of the block Hamiltonian to form $B$, the $m$ most probable states of a block immersed in an environment are used [46]. This leads to disappointing results for the ITF model, it being concluded that the DMRG offered no special advantage over the RSRG when applied to critical phenomena.

The studies in Ref. [46] cannot, however, be considered exhaustive as the block sizes used were quite small. In Ref. [47], it was proposed that the robustness, portability and systematic improvability of the DMRG could be applied to the generation of RG transformations (20) with large block sizes $b$, using the fact that accurate representations of important states can be gleaned for large blocks. 


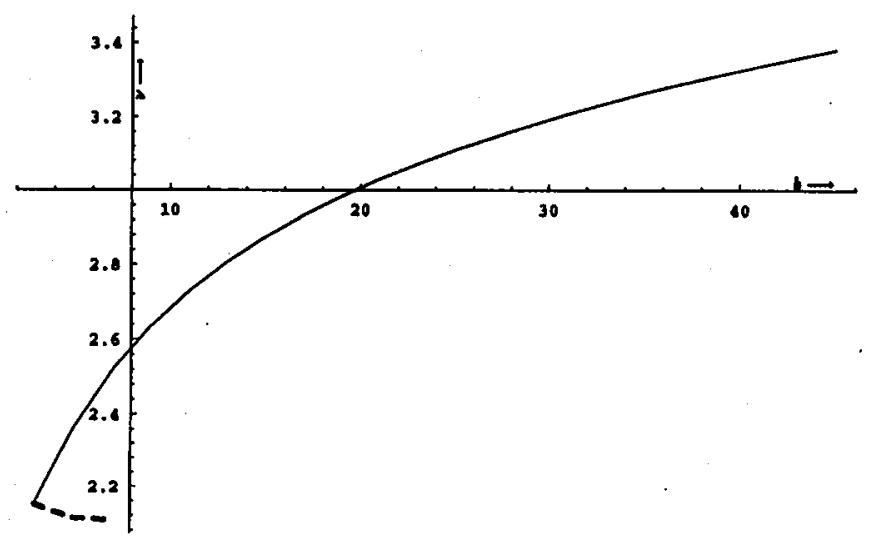

Fig. 3. DMRG result for the critical exponent $\nu$ as a function of block size $b$ for the anisotropic Heisenberg model. The dashed line is the result from the RSRG approach.

This approach was applied to the $X X Z$ model. The result for $\nu$, given as a function of $b$ in Fig. 3, shows a marked improvement over the RSRG result, the essential singularity being recovered in the limit $b \rightarrow \infty$ [47]. It would seem that more testing of the DMRG as a generator of RG transformations might be worthwhile.

Finally, it was noted in [47] that the values of the important eigenvalues in the various symmetry sectors of the density matrix provide a general and intuitive scheme for choosing a suitable value of $m$ and hence for identifying the renormalised Hamiltonian $\mathcal{H}^{\prime}$. For instance, it was noted that the $S=1$ Heisenberg model mapped naturally onto a ladder model under this scheme, as has been demonstrated analytically and numerically [48].

\section{Recent extensions}

In this section we discuss some current trends in advancing the applicability of the DMRG. We focus on recent extensions of the DMRG to the calculation of thermodynamical properties of 2D classical systems and (hence) 1D quantum systems, as well as to the calculation of low energy properties of quantum models in $2 \mathrm{D}$.

\section{1. $2 D$ classical and $1 D$ quantum systems at non-zero temperature}

In a recent paper, Nishino exploited the similarities between the transfer matrix of a 2D classical system and the Hamiltonian of a $1 \mathrm{D}$ quantum system in order to formulate the DMRG for 2D classical systems [49]. Results for the specific heat of the 2D Ising model are most promising, the exact result being recovered with good accuracy over the whole temperature range, even at criticality, using a reasonably modest basis set [49].

Potential applications of this approach include the determination of the critical properties of 2D classical models with high spin, anisotropy and frustration. Also, this extension paves the way for the study of quantum spin chains at 
non-zero temperature, as the Trotter-Suzuki decomposition allows such systems to be mapped onto classical 2D systems.

This programme has been carried out for the dimerised $X Y$ chain

$$
\mathcal{H}=-\sum_{i}\left(S_{2 i-1}^{x} S_{2 i}^{x}+S_{2 i-1}^{y} S_{2 i}^{y}\right)-\gamma \sum_{i}\left(S_{2 i}^{x} S_{2 i+1}^{x}+S_{2 i}^{y} S_{2 i+1}^{y}\right)
$$

Although technical difficulties with the transfer matrix limit the basis sizes which can be used, very reasonable results, down to low temperatures $T$ have been obtained for the internal energy $u$, especially for $\gamma \neq 1$, when the model possesses a substantial gap [50]. A plot of $u$ versus $T$, for $\gamma=2$ is given in Fig. 4.

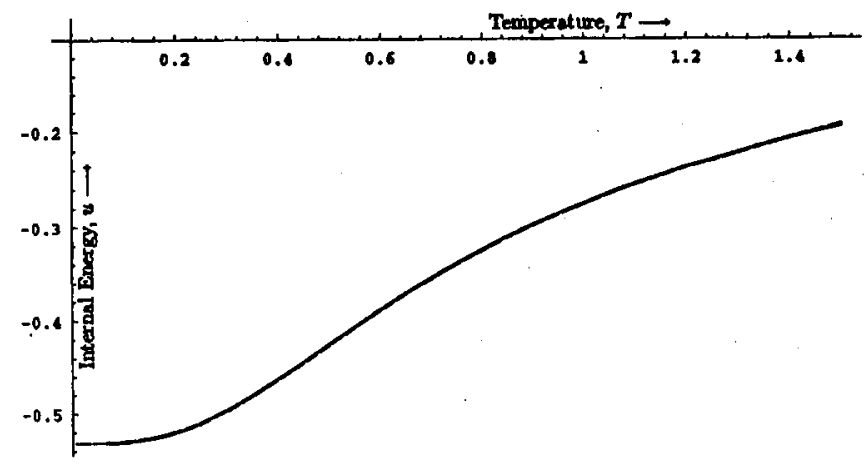

Fig. 4. DMRG result (dashed line) for the internal energy as a function of temperature for the dimerised $X Y$ model with dimerization ratio $\gamma=2$. The full line is the exact result.

Finally, Nishino and Okunishi have derived two further reformulations of the DMRG - the product wave function renormalization group (PWFRG) [51] and the corner transfer matrix renormalization group (CTMRG) [52]. These formulations may offer dramatically improved efficiency over the DMRG and the means of calculating dynamical correlation functions in spin chains [52]. The computational efficiency of the CTMRG has been exploited so as to obtain highly accurate results for the 2D Ising model at criticality [52].

\subsection{Two-dimensional quantum systems}

Soon after the inception of the DMRG, attempts were made at generalising the finite lattice algorithm to two dimensions [53,54]. However, various conditions which permit the unprecedented accuracy of the DMRG in $1 \mathrm{D}$ - the fact that, for short ranged interactions there is no interaction between system and environment blocks, as well as the preservation of reflection symmetry, do not persist in higher dimensions. This is reflected in the relatively slow convergence of the DMRG when applied without modification to two-dimensional systems [53].

In order to circumvent this problem, the DMRG has been reformulated for fermion systems in terms of momentum space basis states [55]. This advancement is made possible through an elegant solution to the technical problem of storing an 
updating potential energy operator which is a sum of products of four creation and annihilation operators. The formulation offers the following distinct advantages:

1. The total momentum can be used as a quantum number in diagonalising the superblock Hamiltonian.

2. Rotational symmetry is not broken. The breaking of this symmetry is a major cause of the slow convergence of the real space formulation of the DMRG.

3. A single program structure applies quite generally in all dimensions.

Results for the 2D Hubbard model are extremely promising [55]. Agreement with exact calculations on $4 \times 4$ lattices is good as is agreement with projected quantum Monte Carlo (PQMC) and stochastic diagonalization (SQ) data for $8 . \times 8$ lattices. In fact, the DMRG, being a truncated basis expansion, generates a variational bound for the ground state energy. This bound is better than that obtained from SQ and is thus the best variational bound on the ground state energy to date (see Table). Also, a numerical solution of the Hubbard model on a $12 \times 12$ lattice has been afforded by this scheme [55].

\section{TABLE}

Comparison of DMRG and SQ results for the ground state energy of the 2D Hubbard model on an $8 \times 8$ lattice, with $U=4 t$ in the usual notation, for various electron fillings $N$.

\begin{tabular}{c|c|c}
\hline \hline$N$ & DMRG & $\mathrm{SQ}^{a}$ \\
\hline 10 & -34.325 & -34.31 \\
18 & -54.394 & -54.37 \\
26 & -66.098 & -66.05 \\
\hline \multicolumn{2}{c}{${ }^{a}$ Ref. [56]. }
\end{tabular}

Potential applications of this approach include heavily doped fermion systems, fermion systems with weak coupling and fermion systems with long-range Coulomb interactions.

Unfortunately, spin Hamiltonians or models with dressed fermion operators such as the $t-J$ model cannot be conveniently expressed in terms of momentum space operators. However, there have been algorithmic advancements in the DMRG which have been exploited to permit the study of such models in 2D [57-59]. White has performed successful calculations of the spin gap in a (frustrated) 2D Heisenberg model for the spin-gapped system $\mathrm{CaV}_{4} \mathrm{O}_{9}$ using a $24 \times 11$ lattice [57] and White and Scalapino have investigated the nature of one and two hole ground states in the $t-J$ model on $10 \times 7$ clusters [58].

These calculations have been made possible by the use of highly efficient code which minimizes the number of Hamiltonian matrix multiplications required in the sparse diagonalization. Moreover, the problem of symmetry breaking which leads to the need for a large number of matrix multiplications has been recently addressed [59]. 
In [59] an optimal scheme for sweeping the lattice has been developed. This has afforded the study of a frustrated Heisenberg model (i.e. with next-neighbour exchange) on a $20 \times 20$ lattice. Good convergence along with the accurate recovery of exact results on the $4 \times 4$ lattice permits confident characterizations of the phases for various values of the coupling constants.

\section{Acknowledgment}

R.J.B. gratefully acknowledges the support of SERC grant no. GR/J26748.

\section{References}

[1] F.D.M. Haldane, Phys. Rev. Lett. 50, 1153 (1983); Phys. Lett. A 93, 464 (1983).

[2] K. Wilson, Rev. Mod. Phys. 47, 773 (1975).

[3] Th. Niemeijer, J.M.J. van Leeuwen, Phys. Rev. Lett. 31, 1411 (1973); Th. Niemeijer, J.M.J. van Leeuwen, Physica 71, 17 (1973).

[4] J.W. Bray, S.T. Chui, Phys. Rev. B 19, 4876 (1979).

[5] S.R. White, R.M. Noack, Phys. Rev. Lett. 68, 3487 (1992); S.R. White, Phys. Rev. Lett. 69, 2863 (1992), Phys. Rev. B 48, 10345 (1993).

[6] S.R. White, D.A. Huse, Phys. Rev. B 48, 3844 (1993); E.S. Sørensen, I. Affleck, Phys. Rev. Lett. 71, 1633 (1993); E.S. Sørensen, I. Affeck, Phys. Rev. B 49, 13235 (1994); E.S. Sørensen, I. Affleck, Phys. Rev. B 49, 15771 (1994).

[7] S. Östlund, S. Rommer, Phys. Rev. Lett. 75, 3537 (1995).

[8] U. Schollwöck, Th. Jolicoeur, Europhys. Lett. 30, 493 (1995); Y. Nishiyama, K. Totsuka, N. Hatano, M. Suzuki, J. Phys. Soc. Jap. 64, 414 (1995).

[9] K. Hallberg, X.Q.G. Wang, P. Horsch, A. Moreo, to be published.

[10] R.J. Bursill, T. Xiang, G.A. Gehring, J. Phys. A 28, 2109 (1994).

[11] U. Schollwöck, Th. Jolicoeur, T. Garel, Phys. Rev. B 53, 3304 (1996).

[12] R.J. Bursill, G.A. Gehring, D.J.J. Farnell, J.B. Parkinson, Tao Xiang, Chen Zeng, J. Phys. C 7, 8605 (1995).

[13] R. Chitra, S. Pati, H.R. Krishnamurthy, D. Sen, S. Ramasesha, Phys. Rev. B 52, 6581 (1995).

[14] Y. Kato, A. Tanaka, J. Phys. Soc. Jap. 63, 1277 (1994).

[15] S. Pati, R. Chitra, D. Sen, H.R. Krishnamurthy, S. Ramasesha, Europhys. Lett. 33, 707 (1996).

[16] M. Azzouz, L. Chen, S. Moukouri, Phys. Rev. B 50, 6223 (1994); S.R. White, R.M. Noack, D.J. Scalapino, Phys. Rev. Lett. 73, 886 (1994); K. Hida, J. Phys. Soc. Jap. 64, 4896 (1995); T. Narushima, T. Nakamura, S. Takada, J. Phys. Soc. Jap. 64, 4322 (1995); U. Schollwöck, D. Ko, Phys. Rev. B 53, 240 (1996).

[17] L. Chen, S. Moukouri, Phys. Rev. B 53, 1866 (1996); S. Moukouri, L. Chen, L.G. Caron, Phys. Rev. B 53, R488 (1996).

[18] S.J. Qin, S.D. Liang, Z.B. Su, L.Yu, Phys. Rev. B 52, R5475 (1995).

[19] C.C. Yu, S.R. White, Phys. Rev. Lett. 71, 3866 (1993); C.C. Yu, S.R. White, Physica $B$ 199, 454 (1994); M. Guerrero, C.C. Yu, Phys. Rev. B 51, 10301 (1995).

[20] S. Moukouri, L.G. Caron, Phys. Rev. B 52, 15723 (1995); N. Shibata, T. Nishino, K. Veda, C. Ishii, Phys. Rev. B 53, R8828 (1996). 
[21] M. Guerrero, R.M. Noack, Phys. Rev. B 53, 3707 (1996).

[22] H. Otsuka; T. Nishino, Phys. Rev. B 52, 15066 (1995); S. Moukouri, L.G. Caron, C. Bourbonnais, L. Hubert, Phys. Rev. B 51, 15920 (1995).

[23] R.M. Noack, S.R. White, D.J. Scalapino, Phys. Rev. Lett. 73, 882 (1994); S.R. White, R.M. Noack, D.J. Scalapino, J. Low Temp. Phys. 99, 593 (1995): R.M. Noack, S.R. White, D.J. Scalapino, Europhys. Lett. 30, 163 (1995); C.A. Hayward, D. Poilblanc, R.M. Noack, D.J. Scalapino, W. Hanke, Phys. Rev. Lett. 75, 926 (1995).

[24] T.A. Costi, P. Schmitteckert, J. Kroha, P. Wölfle, Phys. Rev. Lett. 73, 1275 (1994); S. Eggert, I. Affleck, Phys. Rev. Lett. 75, 934 (1995); E.S. Sørensen, I. Affleck, Phys. Rev. B 51, 16115 (1995); X.Q. Wang, S. Mallwitz, Phys. Rev. B 53, R492 (1996); W. Wang, S.J. Qin, Z.Y. Lu, L. Yu, Z.B. Su, Phys. Rev. B 53, 40 (1996); C.C. Yu, M. Guerrero, to be published.

[25] K. Hida, J. Phys. Soc. Jap. 65, 895 (1996).

[26] P. Schmitteckert, U. Eckern, Phys. Rev. B 53, 15397 (1996).

[27]'K.A. Hallberg, P. Horsch, G. Martinez, Phys. Rev. B 52, R719 (1995).

[28] S.J. Qin,. T.K. Ng, Z.B. Su, Phys. Rev. B 52, 12844 (1995).

[29] H.B. Pang, H. Akhlaghpour, M. Jarrell, Phys. Rev. B 53, 5086 (1996).

[30] K.A. Hallberg, Phys. Rev. B 52, R9827 (i995).

[31] S.K. Pati, S. Ramasesha, Z. Shuai, J.L. Bredas, to be published.

[32] L.G. Caron, S. Moukouri, Phys. Rev. Lett. 76, 4050 (1996).

[33] K. Majumda, D.K. Ghosh, J. Math. Phys. 10, 1388 (1969a); ibid., p. 1399.

[34] G. Castilla, S. Chakravarty, V.J. Emery, Phys. Rev. Lett. 75, 1823 (1995).

[35] S.R. White, I. Affleck, to be published.

[36] C.K. Lai, J. Math. Phys. 15, 1675 (1974); B. Sutherland, Phys. Rev. B 12, 3795 (1975).

[37] K. Okamoto, K. Nomura, Phys. Lett. A 169, 433 (1992); K. Nomura, K. Okamoto, J. Phys. Soc. Jap. 62, 1123 (1993); K. Nomura, K. Okamoto, J. Phys. A 27, 5773 (1994); S. Eggert, to be published.

[38] G. Fath, J. Solyom, Phys. Rev. B 47, 872 (1993).

[39] T. Xiang, G.A. Gehring, J. Magn. Magn. Mater. 104-107, 861 (1992); T. Xiang, G.A. Gehring, Phys. Rev. B 48, 303 (1993).

[40] The Many Body Problem - An Encyclopedia of Exactly Solvable Models in One Dimension, Eds. E. Lieb, D. Mattis, World Scientific, Singapore 1993.

[41] I. Affleck, T. Kenedy, E.H. Lieb, H. Tasaki, Phys. Rev. Lett. 59, 799 (1987).

[42] R.J. Bursill, F. Gode, G.A. Gehring, in preparation.

[43] P. Pfeuty, R. Jullien, K.A. Penson, in: Topics in Current Physics 30, Eds. T.W. Burkhardt, J.M.J. van Leeuwen, Springer-Verlag, Berlin 1982.

[44] S.K. Sarker, Phys. Rev. B 30, 2752 (1984).

[45] G. Spronken, R. Jullien, M. Avignon, Phys. Rev. B 24, 53,56 (1981).

[46] A. Drzewiński, J.M.J. van Leeuwen, Phys. Rev. B 49, 403 (1994); A. Drzewiński, F. Daerden, J. Magn. Magn. Mater. 140-4, 1623 (1995); A. Drzewiński, R. Dekeyser, Phys. Rev. B 51, 15218 (1995).

[47] R.J. Bursill, F. Gode, J. Phys. C 7, 9765 (1995). 
[48] S.R. White, Phys. Rev. B 53, 52 (1996).

[49] T. Nishino, J. Phys. Soc. Jap. 64, 3598 (1995); T. Nishino, K. Okunishi, M. Kikuchi, Phys. Lett. A 213, 69 (1996).

[50] R.J. Bursill, T. Xiang, G.A. Gehring, to appear in J. Phys. C.

[51] T. Nishino, K. Okunishi, J. Phys. Soc. Jap. 64, 4084 (1995).

[52] T. Nishino, K. Okunishi, J. Phys. Soc. Jap. 65, 891 (1996).

[53] S.D. Liang, H.B. Pang, Phys. Rev. B 49, 9214 (1994).

[54] S. Liang, H. Pang, Europhys. Lett. 32, 173 (1995).

[55] T. Xiang, Phys. Rev. B 53, 10445 (1996).

[56] H. Deraedt, M. Frick, Phys. Rep. 231, 107 (1993).

[57] S.R. White, to be published.

[58] S.R. White, D.J. Scalapino, to be published.

[59] T. Xiang, in preparation. 